\title{
DETERMINATION OF MOTOR MOBILITY SPEED ZONES FOR PLAYERS FROM AGE GROUPS U16 AND U17
}

\author{
V. Tsvetkov, M. Gadev, Peter Peev \\ Keywords: junior football, speed, speed zones, GPS;
}

\begin{abstract}
Introduction
As is known, the football game falls into the group of poly-structural sports. The motor activity in this sport discipline is defined as varied and variable intensity.
\end{abstract}

There are many studies in the field of football. The public review of literary sources reveals dozens of editions related to the specificity and methodology of the preparation. All this experience is mostly related to the profile of male and female football, and the child-teen period of sports improvement remains relatively intact from a scientific point of view. Here is a big aspect of child-teen football, which reveals opportunities for improvement and development - defining the profile of the account for a certain age period.

Knowledge in this direction would help to improve the qualitative and quantitative improvement of the players' training during this period of their sport-technical improvement.

What is more, the optimization of the sport-pedagogical process by adequately defining the account profile in the respective age period enables us to implement modern technologies in the training process. For example, we can take ever-wider use of GPS-based load tracking systems. In short, to base absolute data accuracy on GPS systems to improve the conditional training of competitors, you must first be sure that the profile of the individual movements during the football game is clear.

It is these considerations that lead to the need to explore the various forms of movement of the players during the game.

\footnotetext{
Aim and objectives of the study

All of this also dictates the purpose of our research, namely - the determination of the speed zones of the motorized activity in the youth football for the U16-17 age range.
}

From the so-called goal, two main tasks emerge from the realization of the study:

1. Investigation of the literary sources on the problem of the speed zones and the methodology for their determination.

2. Determine their boundaries for the specific age range using an affordable and adequate test battery.

\section{Methods}

In the course of the study, for the solving of the problems we focused on the review of the literary sources regarding the methods for determination of speed zones in football.

As we can see from Carling and Colleagues (2008) - there are a variety of methods for determining the speed zones:

Goto and Team (2015a), Goto and Team (2015b) offer the use of a $5 \mathrm{~m}$ flying start with $5 \mathrm{~m}$ reinforcement. Based on the determination of the average travel speed of this segment, five working areas are defined, each of which is calculated on the basis of the standard deviation: first zone -2 standard deviations; second zone -1 standard deviations; third zone average speed; fourth zone +1 standard deviations and fifth zone +2 standard deviations.

Mendez-Villanueva and Team (2012) offer a totally different approach to determining the areas, namely the basis of the $10 \mathrm{~m}$ flying start test, measured over a $40 \mathrm{~m}$ track and the Vamevall test, calculates the maximum anaerobic and maximum aerobic velocity and the reserve anaerobic velocity, after which 5 working zones of activity are determined. Harley and colleagues (2012) suggests using the following equation (Vpeak SNR / Vpeak GRP) * TH-S to identify 6 travel speeds that are standing, walking, jogging, running, high-speed running and pattern spraying proposed by Bradley and colleagues (2009). Measurements are made on the basis of a $10 \mathrm{~m}$ flying start, taken from a $20 \mathrm{~m}$ run. 
Brendan Chaplin (Meeting of the Minds Conference - 2017) offers a reverse approach to exploring the areas of motor activity. It recommends the use of GPS systems to analyze the speed of each individual movement in a racing environment and then analyze and distribute the movements in five areas - standing, low-intensity, high-intensity, sprint, re-sprint.

The variety of approaches and methods for identifying areas of motor activity lead to numerous contradictions. For the purpose of our study, we refer to the experience of Abt et al. (2009), which presents the need to use different mobility areas for each age range. Taking into account the trends in the development of football game preparation, it should be noted that although some authors find differences in motor mobility in individual posts (Molinos, 2013, Bradley and colleagues, 2009, Bloomfield and colleagues, 2007, Buchheit and colleagues, 2010), we find it inappropriate for a collective game to individualize the workload in posts. Moreover, the practice of individualizing the workload in male and female football is not included in the training programs (source - FIFA), which gives us grounds to reject such assumptions as irrelevant for the purpose of the study.

Going to the real part of the study, we used the method of sport-pedagogical testing. Taking into consideration the experience of previous research mentioned in the literature, we have approached the development of its methodology and model for the determination of mobility mobility speed ranges for 16-17 year old players. It is based on specially selected tests for compliance with the major mobility speed zones during an official match.

We have identified five speed zones:

3. I speed - walking;

4. II speed - low-intensity running (light running);

5. III Speed - Medium-Speed Journey (Temporal Journey);

6. IV speed - high-intensity running (speed endurance encountered in literary sources such as $R S A$ - Repeated Sprint Ability)

7. $V$ speed - sprint (maximum speed); Taking into account these speeds, we have also selected the appropriate tests. Their choice is dictated by the five areas of energy supply - aerobic, aerobic-anaerobic, anaerobic-aerobic, anaerobic glycolytic and anaerobic alactate.
To determine the maximum walking speed, low-intensity running and sprinting, we measured the time that the 16-17 year-old footballers overcome the 20-meter distance. To confirm the choice of the particular stretch are Bangsbo and colleagues (2006) and Thomas Haugen (2015), according to which the average length of sprinters in football is in the order of 10-15 m. According to Chamari Team (2004), studies based on 10m segments are not sufficiently informative.

In terms of walking we will make it clear that it is the first velocity of the main motoring activity in football, although a number of authors also use standing as such (Bradley et al., 2009, Reilly et al., 1976), quoted by Stoyer and colleague, 2004).

In determining $V$-th speed - sprint (maximum speed), we used the test $20 \mathrm{~m}$ from a flying start, providing an area of $10 \mathrm{~m}$ for pre-amplification and $20 \mathrm{~m}$ for the result.

The tests were carried out on artificial grass. The time to overcome the segments was measured using a Newtest Powertimer, Newtest Oy - Finland electronic photo system, with an accuracy of $0.01 \mathrm{~s}$.

The determination of the III-rd speed - medium-intensity jogging was achieved through a 600-meter test of smooth running. It was carried out on a field with artificial grass along a trail marked with cones every $10 \mathrm{~m}$.

The determination of the $I V$-th speed - high-speed jump (RSA) was performed by a $3 \times 50 \mathrm{~m}$ shuttle test. The test was carried out on a football field with artificial grass. It consists of 3 runs of 50 meters, without interruption.

The determination of II-nd speed - low intensity jog, we determined after the result of a given player in the test 600 smooth running, we took the result of the same player for the $20 \mathrm{~m}$ walking test. The resulting difference allowed us to digitally visualize the boundary speeds of this zone.

We processed the data from the field trials using a variation analysis using a statistical program (SPSS Inc. v. 19, Chicago, USA).

In the overall survey participated a contingent of 40 football players aged 16-17 years (2000 and 2001) by the Youth Acadey of PFC "Levski" - Sofia. 


\section{Results and discussion}

The last method we applied to carry out the study is statistical variation analysis. Through it, we ana- lyzed the results obtained, which allowed us to distinguish the speeds determining the five areas of mobility.

\begin{tabular}{|c|c|c|c|c|c|c|c|c|c|}
\hline TEST & $\mathbf{n}$ & $\mathbf{X} \mathbf{~ m i n}$ & $\mathbf{X} \mathbf{m a x}$ & $\mathbf{R}$ & $\mathbf{C}$ & $\mathbf{S}$ & $\mathbf{V}$ & As & Ex \\
\hline 20m walk & 40 & 9.19 & 12.55 & 3.36 & 11.01525 & 0.78 & 7.12 & -0.088 & -0.431 \\
\hline 600m running & 40 & 94.54 & 115.82 & 21.28 & 104.0265 & 5.80 & 5.57 & 0.247 & -1.152 \\
\hline 3x50m shuttle run & 40 & 21.15 & 24.86 & 3.71 & 23.5625 & 0.93 & 3.96 & -0.74 & 0.389 \\
\hline 20m flying start & 40 & 2.38 & 2.75 & 0.37 & 2.563 & 0.11 & 4.17 & 0.091 & -1.235 \\
\hline
\end{tabular}

Table 1

In Table 1, we see the summarized data from the test results. We see that coefficients of variance $(\mathrm{V})$ determine the contingent of the tested individuals as very homogeneous - V\% of 3.96-7.12. From the following follows the conclusion that the sample is statistically reliable.
In order to convert time values into motion velocities, we used the well-known formula which characterizes the relationship between path, velocity and time $-\mathbf{S}=V t$. The normal distribution allows us to use the magnitude $(\mathrm{R})$ as an area that we can claim to represent the corresponding area of mobility. The resulting speed zones are summarized in Table 2.

\begin{tabular}{|c|c|c|c|c|}
\hline \multirow{2}{*}{ Type of movement } & \multicolumn{3}{|c|}{ Speed zones } \\
\cline { 2 - 5 } & \multicolumn{2}{|c|}{$\mathbf{m} / \mathbf{s}$} & \multicolumn{2}{c|}{$\mathbf{~ k m}$} \\
\hline Sprint & above 7.2 & & above 25.9 & \\
\hline High intensity running & 6.06 & 7.1 & 21.8 & 25.8 \\
\hline Medium intensity running & 5.18 & 6.05 & 18.6 & 21.7 \\
\hline Low intensity running & 2.19 & 5.17 & 7.9 & 18.5 \\
\hline Walking & & up to 2.18 & & up to 7.8 \\
\hline
\end{tabular}

Table 2

\section{Conclusions}

1. The methods used so far to distinguish areas of mobility in the childhood period do not carry the necessary amount of information. That is why we cannot fully rely on the existing models when introducing the respective areas into the training process.

2. The methodology we offer is more practical than theoretical, allowing for ease of application in field conditions as well as simpler extraction of the necessary amount of information. It (the methodology) fully reflects the level of physical performance of the team.

3. Due to the proposed model, the problem of using GPS-based load-determination systems at the stage of children's and teen football is solved.

\section{References}

Abt, G. \& R. Lovell (2009), The use of individualized speed and intensity thresholds for determining the distance run at high-intensity in professional soccer, Journal of Sports Sciences, July 2009; 27(9): 893-898

Aguiar, M., C. Abrantes, V. Maçãs, N. Leite1, J. Sampaio \& S. Ibáñez (2008), Effects of intermittent or continuous training on speed, jump and repeated-sprint ability in semi-professional soccer players, The Open Sports Sciences Journal, 1, 15-1915

Anolli, L., S. Duncan Jr., M.S. Magnusson \& G. Riva (2005), The hidden structure of interaction: From neurons to culture patterns, Amsterdam: IOS Press, $283 \mathrm{pp}$ Aughey, Robert J. (2011), Applications of GPS Technologies to Field Sports, International Journal of Sports Physiology and Performance, 6, p. 295-310

Bangsbo, J. (2014), Physiological demands of football, 
Sports Science Exchange, 27(125), 1-6

Bangsbo, J., M. Mohr, \& P. Krustrup (2006). Physical and metabolic demands of training and match-play in the elite football player, Journal of Sports Sciences, 24: 665-674

Bradley, P.S., W. Sheldon, B. Wooster, P. Olsen, P. Boanas \& P. Krustrup (2009), High-intensity running in English FA Premier League Soccer Matches, Journal of sports sciences, January 15;27(2):159-68

Buchheit, M., A. Mendez-Villanueva, B. M. Simpson \& P.C. Bourdon (2010), Match Running Performance and Fitness in Youth Soccer, International Journal of Sports Medicine, Nov;31(11):818-25.

Bundle, M.W., R. W. Hoyt \& P. G. Weyand (2003), Highspeed running performance: a new approachto assessment and prediction, Journal of Applied Physiology 95: $1955-1962$
Casas, A. (2008), Physiology and methodology of intermittent resistance training for acyclic sports, Journal of Human Sport \& Exercise 2008; 3(1), 23-52

Chamari, K., Y. Hachana, Y.B. Ahmed, O. Galy, F. Sghaier, J-C. Chatard, O. Hue \& U. Wisløff (2004), Field and laboratory testing in young elite soccer players, British Journal of Sports Medicine, 38:191-196

Di Salvo, V. R. Baron, H. Tschan, F. J. Calderon Montero, N. Bachl, F. Pigozzi (2007), Performance characteristics according to playing position in elite soccer, International Journal of Sports Medicine, 28: 222-227

Garrett Jr, W.E., D.T. Kirkendall \& S.R. Contiguglia (1996), The US soccer medicine book, Williams and Willkins, $489 \mathrm{pp}$

Goto, H., J. Morris \& M.E. Nevill (2015a), Match Analysis of U9 and U10 English Premier League academy soccer Players using a Global Positioning System, Journal of strength and conditioning research, April; 29(4):954-63 